\title{
Distribuição ambiental e temporal das comunidades de macroalgas de riachos da Serra da Prata, Estado do Paraná, Sul do Brasil
}

\author{
CLETO K. PERES ${ }^{1}$, CIRO C. Z. BRANCO ${ }^{1,2}$ e ROGÉRIO A. KRUPEK ${ }^{1}$
}

(recebido: 28 de fevereiro de 2008; aceito: 08 de julho de 2009)

\begin{abstract}
Environmental and temporal distribution of stream macroalgal communities from Serra da Prata, Paraná State, southern Brazil).Considering the scarcity of studies focusing on stream macroalgal communities in subtropical regions, the present study was conducted to investigate the environmental and temporal distribution patterns of these communities in a well-preserved region of ombrophilous dense forest. Eight samplings sites were sampled twice (Summer and Winter) in the Serra da Prata (SaintHilaire/Lange National Park), located in eastern portion of the Parana State, southern Brazil. The Principal Components Analysis (PCA) showed that the environmental variables of the investigated streams had a clear temporal pattern, with an evident distinction between winter and summer conditions. In contrast, the Detrended Correspondence Analysis (DCA), based on the presence/absence of macroalgae, did not reveal any temporal pattern, without evident differences between winter and summer floristic compositions. One pattern showed by the DCA was the separation of sampling sites by the intensity of riparian vegetation shading. Overall, the study region showed low values of richness and abundance, and the occurrence of majority of taxa were restricted to a single sampling site. Thus, the results suggested that the structure of the macroalgal communities studied seem to respond to a combination of environmental variables that change continuously in time and space, emphasizing the importance, among others, of variations on a small scale (microhabitat). Moreover, the results also showed that, on a global scale, the distribution of lotic macroalgae in the study region can, apparently, be limited by the degree of shading imposed by riparian vegetation.
\end{abstract}

Key-words - local variation, ombrophilous dense forest, Saint-Hilaire/Lange National Park, shading, subtropical region

RESUMO - (Distribuição ambiental e temporal das comunidades de macroalgas de riachos da Serra da Prata, Estado do Paraná, Sul do Brasil). Considerando a carência de estudos enfocando comunidades de macroalgas de ambientes lóticos em regiões subtropicais, o presente estudo foi conduzido com o objetivo de investigar o padrão de distribuição destas comunidades em uma região de floresta ombrófila densa bem preservada. Oito pontos de amostragem foram amostrados em duas estações contrastantes (inverno e verão) na Serra da Prata (Parque Nacional Saint-Hilaire/Lange), localizada na porção leste do Estado do Paraná, Sul do Brasil. A Análise dos Componentes Principais (PCA) mostrou que as variáveis ambientais dos riachos analisados tiveram um padrão temporal claro, com uma distinção evidente entre inverno e verão. De modo contrário, a Análise de Correspondência Destendenciada (DCA), baseada na presença/ausência de espécies, não revelou nenhum padrão temporal ou diferenças entre a composição florística do inverno e do verão. Um padrão mostrado na DCA foi a separação dos pontos de amostragem pela intensidade do sombreamento da vegetação ripária. De maneira geral, a região estudada apresentou baixos valores de riqueza e abundância, e a maioria dos táxons foi restrita a um único ponto ou época de amostragem. Deste modo, os resultados sugerem que a estruturação das comunidades de macroalgas estudadas parecem responder à combinação das variáveis ambientais que se alteram continuamente no tempo e no espaço, enfatizando, entre outras, a importância das variações em pequena escala (microhabitat). Por outro lado, os resultados também indicaram que, em escala global, a distribuição das macroalgas dos ambientes lóticos da região de estudo, aparentemente, pode ser limitada pelo grau de sombreamento imposto pela vegetação marginal.

Palavras chave - floresta ombrófila densa, Parque Nacional Saint-Hilaire/Lange, região subtropical, sombreamento, variação local

\section{Introdução}

Nas últimas décadas vários estudos têm sido conduzidos em todo o mundo enfocando a ecologia de organismos de ambientes lóticos (Allan 1995), sendo a mesma tendência observada para macroalgas (Sheath \& Burkholder 1985, Entwisle 1990, Sheath \& Cole 1992, Pfister 1993, Leukart 1995, Hu \& Xie 2006). Na região Sudeste do Brasil, em particular, um importante conjunto

1. Universidade Estadual Paulista, Faculdade de Ciências e Letras, Departamento de Biologia, Av. Dom Antônio, 2100, 19806-900 Assis, SP, Brasil.

2._Autor para correspondência: czbranco@assis.unesp.br de informações a respeito de comunidades de macroalgas lóticas pode ser encontrado na literatura (e.g. Necchi Júnior \& Pascoaloto 1993, Necchi Júnior et al. 1994, 2000, 2003, 2008, Branco \& Necchi Júnior 1996, 1998). Entretanto, na região subtropical brasileira pouco se conhece sobre a distribuição ecológica das comunidades de macroalgas. As informações disponíveis até o momento abordando tais aspectos são encontradas em Krupek et al. (2007) e Branco et al. (2008, 2009), para as regiões centro-sul e centro-oeste do Estado do Paraná, respectivamente.

Entre os padrões de distribuição ambiental descritos para comunidades de macroalgas de ambientes lóticos pode ser destacada a distribuição em mosaico 
caracterizada pela correlação direta entre riqueza e abundância de espécies (Borges \& Necchi Júnior 2006). A recorrência de tal padrão em diferentes tipos de riachos de diversas partes do mundo permite sugerir o mesmo como sendo universal para tais comunidades (Sheath \& Burkholder 1985, Sheath \& Cole 1992, Branco \& Necchi Júnior 1996, 1998, Necchi Júnior et al. 2000, 2003, Hu \& Xie 2006). Outro padrão que também tem sido freqüentemente reportado é a alta proporção de espécies de ocorrência restrita a somente um ou poucos riachos, mesmo em estudos envolvendo áreas amostrais de dimensões regionais ou continentais (Hu \& Xie 2006, Krupek et al. 2007, Branco et al. 2009).

De modo geral, estudos sugerem que a estruturação dos padrões de distribuição ambiental destas comunidades está relacionada a determinadas variáveis ambientais isoladas ou em conjunto (e.g., $\mathrm{pH}$ - Sheath \& Burkholder 1985, temperatura - Sheath et al. 1986, substrato - Sheath \& Müller 1997, precipitação pluviométrica - Necchi Júnior \& Pascoaloto 1993, Branco \& Necchi Júnior 1998). Entretanto, a falta de uma relação direta e evidente entre a estrutura das comunidades de macroalgas com tais variáveis ambientais também têm sido registrada na literatura (Krupek et al. 2007, Branco et al. 2009).

A necessidade de avaliar os padrões de distribuição ambiental das comunidades de macroalgas lóticas e o papel das variáveis (ou falta dele) que os determinam impõe a ampliação dos estudos em regiões geográficas distintas e pouco estudadas. Considerando que os padrões de distribuição ecológica de tais comunidades são muito pouco conhecidos na região subtropical do Brasil, o presente trabalho foi desenvolvido na Serra da Prata, em área de floresta ombrófila densa em excelente estado de conservação, localizada na porção leste do Estado do Paraná, Brasil.

\section{Material e métodos}

A Serra da Prata está localizada entre as longitudes $48^{\circ} 33^{\prime} \mathrm{W}$ e $48^{\circ} 43^{\prime} \mathrm{W}$ e entre as latitudes $25^{\circ} 32^{\prime} \mathrm{S}$ e $25^{\circ} 51^{\prime} \mathrm{S}$. A área de estudo está quase totalmente inserida no Parque Nacional Saint-Hilaire/Lange, o qual possui uma área de aproximadamente 25.000 hectares localizada entre os municípios de Matinhos, Guaratuba, Morretes e Paranaguá. A vegetação encontrada na Serra da Prata é composta basicamente pela Floresta Ombrófila Densa (Roderjan et al. 2002).

O clima na porção leste do Estado do Paraná é influenciado diretamente pelas massas de ar quente e úmido do Oceano Atlântico, sendo enquadrado na categoria Af $(t)$ de Köeppen (Maack 1981, Roderjan et al. 2002). Nesta região não há estação seca e as precipitações médias anuais podem exceder 3.000 mm (Pró-Atlântica 2002).
Com base em estimativas de estudos previamente realizados (Branco \& Necchi Júnior 1996, Necchi Júnior et al. 2000,2003 ) e na área total da Serra da Prata (aproximadamente 25.000 hectares) foram amostrados oito segmentos de riachos (tabela 1), em duas estações contrastantes do ano (julho/2005 - inverno e, fevereiro/2006 - verão), através da técnica da transeção (Necchi Júnior et al. 1994, 2000, 2003). A riqueza e a abundância foram estimadas através da análise visual (Necchi Júnior 1993), uma técnica amplamente utilizada em estudos com macroalgas lóticas (Necchi Júnior et al. 1994, 2000, 2003, 2008, Hu \& Xie 2006, Krupek et al. 2007, Branco et al. 2009) e que apresenta resultados comparáveis a outras técnicas como peso seco e clorofila $a$ (Necchi et al. 1995).

Variáveis físicas e químicas selecionadas (temperatura, turbidez, condutividade específica, $\mathrm{pH}$ e oxigênio dissolvido) foram medidas diretamente em campo com um controlador de qualidade da água Horiba U-10. Ainda, a velocidade da correnteza foi medida utilizando-se fluxômetro mecânico General Oceanics 2030R, e a profundidade foi estimada com uma régua. Foram anotados também o tipo de substrato (de acordo com o tamanho de partículas de Gordon et al. 1992) e o grau de sombreamento (utilizando-se as classes de sombreamento aberto, parcialmente sombreado, sombreado e fortemente sombreado de DeNicola et al. 1992).

Dados sobre a precipitação acumulada nos últimos 7, 15 e 30 dias anteriores a amostragem foram obtidos na estação pluviométrica do Instituto Agronômico do Paraná (Iapar) de Morretes - PR, localizada a aproximadamente cinco quilômetros do local de estudos. Esses dados são importantes

Tabela 1. Localização dos pontos de amostragem na Serra da Prata, porção leste do Estado do Paraná.

Table 1. Location of the sampling sites in the Serra da Prata, eastern portion of the Paraná State.

\begin{tabular}{|c|c|}
\hline Ponto & Localização \\
\hline 1 & $\begin{array}{l}\text { Município de Matinhos, PR, cachoeira do Tigre } \\
\text { - rio Cachoeira. } 25^{\circ} 44^{\prime} 27^{\prime \prime} \mathrm{S}, 48^{\circ} 36^{\prime} 66^{\prime \prime} \mathrm{W} \text {. }\end{array}$ \\
\hline 2 & $\begin{array}{l}\text { Município de Matinhos, PR, riacho próximo } \\
\text { ao Pesque-pague das Tilápias. } 25^{\circ} 44^{\prime} 80^{\prime \prime} \mathrm{S} \text {, } \\
48^{\circ} 35^{\prime} 92^{\prime \prime} \mathrm{W} \text {. }\end{array}$ \\
\hline 3 & $\begin{array}{l}\text { Município de Matinhos, PR, rio Cambará. } \\
25^{\circ} 43^{\prime} 53^{\prime \prime} \mathrm{S}, 48^{\circ} 35^{\prime} 86^{\prime} \mathrm{W} \text {. }\end{array}$ \\
\hline 4 & $\begin{array}{l}\text { Município de Paranaguá, PR, rio da Colônia } \\
\text { Pereira. } 25^{\circ} 41^{\prime} 51^{\prime \prime} \mathrm{S}, 48^{\circ} 35^{\prime} 14^{\prime \prime} \mathrm{W} \text {. }\end{array}$ \\
\hline 5 & $\begin{array}{l}\text { Município de Paranaguá, PR, rio das Pombas. } \\
25^{\circ} 39^{\prime} 18^{\prime \prime} \text { S, } 48^{\circ} 35^{\prime} 42^{\prime \prime} \mathrm{W} \text {. }\end{array}$ \\
\hline 6 & $\begin{array}{l}\text { Município de Paranaguá, PR, rio da Colônia Maria } \\
\text { Luisa. } 25^{\circ} 38^{\prime} 63^{\prime \prime} \mathrm{S}, 48^{\circ} 36^{\prime} 05^{\prime \prime} \mathrm{W} \text {. }\end{array}$ \\
\hline 7 & $\begin{array}{l}\text { Município de Paranaguá, PR, riacho do Morro } \\
\text { Inglês. } 25^{\circ} 36^{\prime} 24^{\prime \prime} \mathrm{S}, 48^{\circ} 37^{\prime} 66^{\prime \prime} \mathrm{W} \text {. }\end{array}$ \\
\hline 8 & $\begin{array}{l}\text { Município de Matinhos, PR, rio Indaial. } \\
25^{\circ} 47^{\prime} 79^{\prime \prime} \mathrm{S}, 48^{\circ} 34^{\prime} 01^{\prime} \mathrm{W} \text {. }\end{array}$ \\
\hline
\end{tabular}


para a visualização de potenciais distúrbios ocasionados pela precipitação, os quais em última análise poderiam afetar a riqueza e abundância das comunidades de macroalgas.

Os dados físicos e químicos da água foram submetidos à Análise dos Componentes Principais (PCA) (Digby \& Kempton 1987), aplicando-se o Coeficiente $r$ de Pearson para avaliar possíveis padrões de distribuição espacial nos diferentes riachos ou estações. O Teste de Wilcoxon (Conover 1971) foi aplicado para verificar a presença de possíveis diferenças temporais entre os valores médios de riqueza e abundância das espécies encontradas e também para discriminar os padrões visualizados na PCA. A Análise de Correspondência Destendenciada (DCA) (McCune \& Mefford 1999) foi aplicada para discriminar padrões de distribuição espacial das macroalgas com base na presença/ ausência de espécies. Finalmente, para avaliar possíveis espécies tipicamente associadas a uma estação do ano específica ou, condição ambiental foi utilizada a Análise de Espécies Indicadoras (ISA) (Dufrêne \& Legendre 1997). Todos os testes acima indicados foram realizados com uso dos pacotes estatísticos Statistica (Statisoft Software) e PC-ORD 4.0 (McCune \& Mefford 1999).

\section{Resultados}

De modo geral, os riachos da Serra da Prata foram caracterizados por ambientes com temperatura $\left(\bar{x}=21,5 \pm 1,4{ }^{\circ} \mathrm{C} ; \mathrm{CV}=0,06\right)$, condutividade específica $\left(\bar{x}=31,5 \pm 5,3 \mu \mathrm{S} \mathrm{cm}^{-1} ; \mathrm{CV}=0,17\right)$ e oxigênio dissolvido $\left(\bar{x}=5,9 \pm 0,6 \mathrm{mg} \mathrm{L}^{-1} ; \mathrm{CV}=0,11\right)$ moderados, $\mathrm{pH}$ $(\bar{x}=6,7 \pm 0,3 ; \mathrm{CV}=0,05)$ neutro a levemente ácido, turbidez $(\bar{x}=1,5 \pm 1,2 \mathrm{NTU} ; \mathrm{CV}=0,81)$ extremamente baixa, velocidade da correnteza $\left(\bar{x}=74 \pm 54 \mathrm{~cm} \mathrm{~s}^{-1}\right.$; $\mathrm{CV}=0,73)$ com variações extremas e baixas profundidades $(\bar{x}=27 \pm 13 \mathrm{~cm} ; \mathrm{CV}=0,45)$. Os valores de precipitação acumulada nos últimos sete, quinze e trinta dias antes de cada amostragem foram muito maiores no verão $(110,190,290 \mathrm{~mm}$, respectivamente) do que no inverno (3, 45, $90 \mathrm{~mm}$ ) (figura 1).

Em relação ao sombreamento, 63\% dos pontos de amostragem foram classificados como fortemente sombreados, e os $37 \%$ restantes foram classificados como sombreados. Quanto ao substrato, houve predomínio dos tipos rocha contínua e matacão, sendo que em $81 \%$ dos segmentos amostrados mais do que 50\% do leito foi dominado por estes tipos. Outras classes de substrato tais como areia, cascalho, seixos, troncos e macrófitas também foram registrados em menor proporção.

O levantamento florístico resultou na identificação de 14 táxons distribuídos em quatro divisões (tabela 2). $\mathrm{O}$ número de táxons em cada ponto por amostragem apresentou valores baixos tanto no inverno $(\bar{x}=1,8)$,

Tabela 2. Ocorrência dos táxons de macroalgas por ponto de amostragem e estação na Serra da Prata, porção leste do Estado do Paraná.

Table 2. Occurrence of macroalgal taxa per sampling sites and season in the Serra da Prata, eastern portion of the Paraná State.

\begin{tabular}{lcc}
\hline \multirow{2}{*}{ Táxons } & \multicolumn{2}{c}{ Pontos de ocorrência nas estações } \\
\cline { 2 - 3 } & Inverno & Verão \\
\hline CYANOPHYTA & - & $4,6,7$ \\
Blennothrix komarekii Branco \& Montejano & $2,5,6,7$ & $2,6,8$ \\
Microcoleus subtorulosus Gomont ex Gomont & 1 & 1 \\
Nostoc verrucosum Vaucher ex Bornet \& Flahault & 4 & - \\
Nostoc sp. & - & 2 \\
Nostochopsis lobatus Wood ex Bornet \& Flahault & 7 & $-6,7,8$ \\
Phormidium aerugineo-caeruleum (Gomont) Anagnostidis \& Komárek & 1 & 6 \\
Phormidium retzii (C. Agardh) Gomont ex Gomont & - & 3 \\
Pleurocapsa fluviatilis Lagerheim & & - \\
CHLOROPHYTA & 1,3 & 3 \\
Ecballocystis pulvinata Bohlin var. pulvinata & 3 & - \\
Spirogyra sp. & - & - \\
Zygnema sp. & & \\
HETEROKONTOPHYTA & 4 & \\
Hydrosera whampöensis (Schwartz) Deby var. whampöensis & & \\
RHODOPHYTA & 5 & \\
Batrachospermum ambiguum Montagne & 8 & \\
Estágio 'Chantransia’ de Batrachospermum sp. & & \\
\hline
\end{tabular}



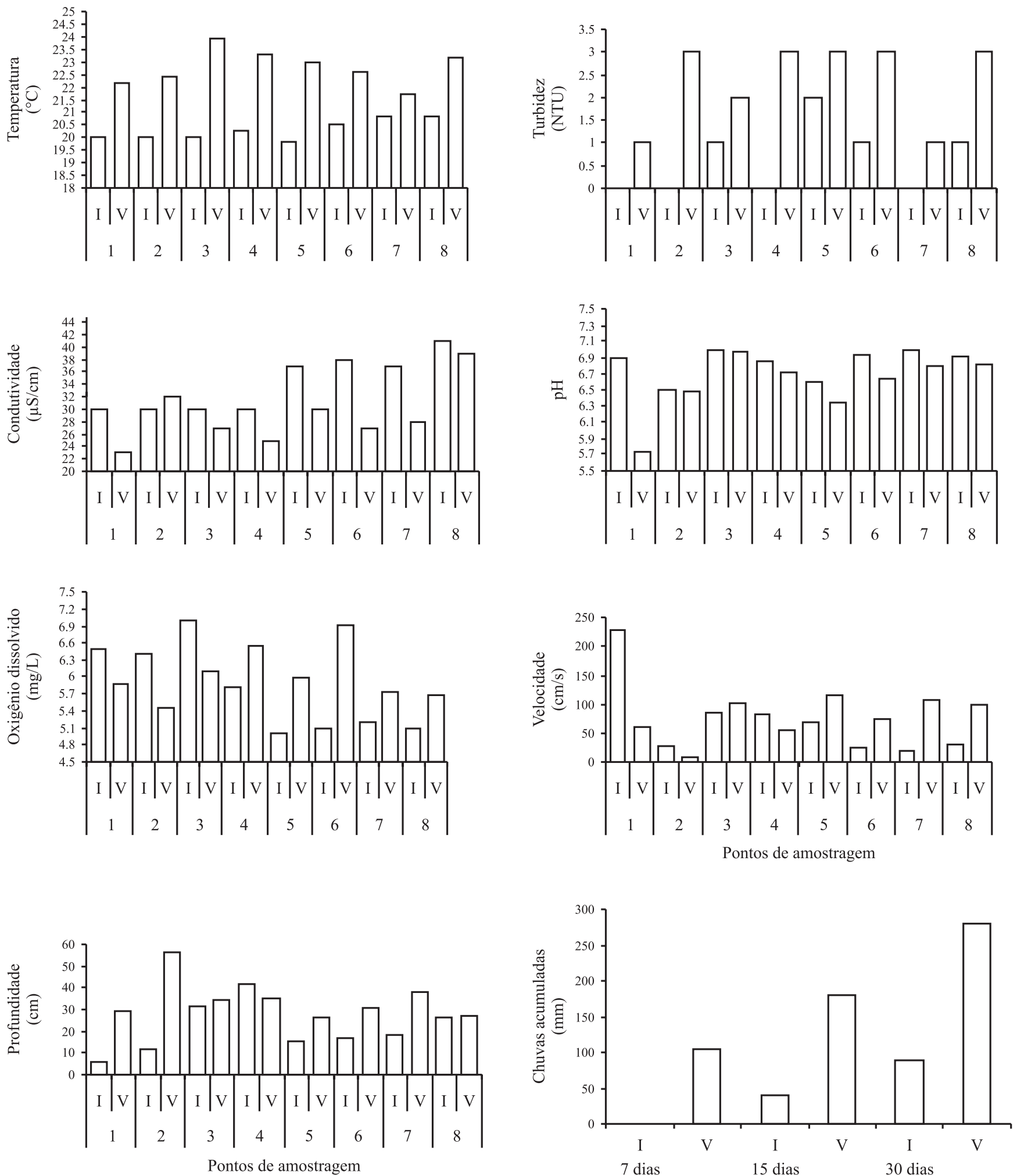

Figura 1. Valores das variáveis físicas e químicas mensuradas nos riachos durante as amostragens do inverno (I) e do verão (V) e, valores de precipitação acumulada nos últimos 7, 15 e 30 dias antes de cada coleta.

Figure 1. Values of the physical and chemical variables measured in the streams during the winter (I) and summer (V) samplings and, precipitation accumulated values in the last 7,15 and 30 days before each sampling. 
quanto no verão $(\bar{x}=2,3)$ e a abundância nunca alcançou $5 \%$ de cobertura percentual $(\bar{x}=1,5)$ (tabela 3 ). Não houve diferença significativa nos valores de riqueza $(z=0,845, P>0,05)$ nem de abundância $(z=1,680$, $P>0,05)$ entre as duas estações contrastantes.
A divisão melhor representada foi Cyanobacteria com 57\% do total de táxons, seguida por Chlorophyta com 22\%, Rhodophyta com $14 \%$ e Heterokontophyta com $7 \%$. As espécies mais amplamente distribuídas foram Microcoleus

Tabela 3. Riqueza e abundância de macroalgas identificadas na Serra da Prata nas duas amostragens (inv = inverno; ver $=$ verão).

Table 3. Richness and abundance of macroalgae identified in the Serra da Prata in the two samplings (inv = winter; ver $=$ summer).

\begin{tabular}{|c|c|c|c|}
\hline \multirow{2}{*}{ Ponto } & \multirow{2}{*}{ Riqueza } & \multicolumn{2}{|r|}{ Abundância } \\
\hline & & Total & Espécies \\
\hline $1 \mathrm{inv}$ & 3 & $1,8 \%$ & $\begin{array}{l}\text { Nostoc verrucosum }(0,8 \%) \text {; Ecballocystis pulvinata var. pulvinata }(0,9 \%) \text {; } \\
\text { Phormidium retzii }(0,1 \%) \text {. }\end{array}$ \\
\hline 2 inv & 1 & $0,3 \%$ & Microcoleus subtorulosus $(0,3 \%)$ \\
\hline 3 inv & 2 & $2,2 \%$ & Ecballocystis pulvinata var. pulvinata $(1,4 \%) ;$ Spirogyra sp. $(0,8 \%)$ \\
\hline 4 inv & 2 & $1,5 \%$ & Hydrosera whampöensis var. whampöensis (0,5\%); Nostoc sp. (1,0\%). \\
\hline $5 \mathrm{inv}$ & 2 & $0,2 \%$ & Microcoleus subtorulosus $(0,1 \%)$; Batrachospermum ambiguum $(0,1 \%)$. \\
\hline 6 inv & 1 & $0,1 \%$ & Microcoleus subtorulosus $(0,1 \%)$ \\
\hline 7 inv & 2 & $1,8 \%$ & Phormidium aerugineo-caeruleum (1,4\%); Microcoleus subtorulosus $(0,4 \%)$. \\
\hline 8 inv & 1 & $0,1 \%$ & Estágio 'Chantransia' $(0,1 \%)$ \\
\hline 1 ver & 1 & $0,1 \%$ & Nostoc verrucosum $(0,1 \%)$ \\
\hline 2 ver & 2 & $0,9 \%$ & Microcoleus subtorulosus $(0,5 \%) ;$ Nostochopsis lobatus $(0,4 \%)$ \\
\hline 3 ver & 3 & $3,7 \%$ & $\begin{array}{l}\text { Ecballocystis pulvinata var. pulvinata }(3,1 \%) \text {; Zygnema sp. }(0,5 \%) \text {; Phormidium } \\
\text { aerugineo-caeruleum }(0,1 \%) \text {. }\end{array}$ \\
\hline 4 ver & 2 & $3,6 \%$ & Phormidium aerugineo-caeruleum (3,5\%); Blennothrix komarekii (0,1\%). \\
\hline 5 ver & 1 & $0,4 \%$ & Estágio 'Chantransia' $(0,4 \%)$ \\
\hline 6 ver & 4 & $2,6 \%$ & $\begin{array}{l}\text { Microcoleus subtorulosus }(0,1 \%) \text {; Phormidium aerugineo-caeruleum }(1,0 \%) \text {; } \\
\text { Blennothrix komarekii }(1,0 \%) ; \text { Pleurocapsa fluviatilis }(0,5 \%) \text {. }\end{array}$ \\
\hline 7 ver & 3 & $2,0 \%$ & $\begin{array}{l}\text { Phormidium aerugineo-caeruleum ( } 0,4 \%) \text {; Estágio 'Chantransia' (1,2\%); } \\
\text { Blennothrix komarekii }(0,4 \%) \text {. }\end{array}$ \\
\hline 8 ver & 2 & $1,0 \%$ & Microcoleus subtorulosus (0,4\%); Phormidium aerugineo-caeruleum (0,6\%). \\
\hline
\end{tabular}

subtorulosus e Phormidium aerugineo-caeruleum que ocorreram em cinco pontos de amostragem cada um. Somente quatro táxons (29\%) ocorreram nas duas estações do ano em um mesmo ponto de amostragem, por outro lado, oito táxons (57\%) ocorreram em apenas um único ponto de amostragem em somente uma estação.

A Análise dos Componentes Principais (PCA) (tabela 4, figura 2) discriminou no Eixo 1 dois grupos de pontos de amostragem. Este eixo foi influenciado principalmente pela condutividade, relacionada com o grupo de pontos com amostragem no inverno e, temperatura e turbidez, relacionadas com o grupo de pontos com amostragem no verão. O Teste de Wilcoxon, realizado com os escores do Eixo 1 da PCA entre os dois grupos de pontos de amostragem discriminados,
Tabela 4. Resultados da Análise de Componentes Principais (PCA) das variáveis físico-químicas e correlação de cada variável com os dois primeiros eixos. (Valores de $r:<0,05^{*}$; $<0,01 * * ;<0,001 * * *)$.

Table 4. Principal Component Analysis results of physical and chemical variables and correlation of each variable with the first two axes. (Values of $r:<0,05^{*} ;<0,01^{* *} ;<0,001^{* * *}$ ).

\begin{tabular}{lcc}
\hline Variáveis/eixos & 1 & 2 \\
\hline Temperatura & $0,833^{* * *}$ & 0,287 \\
Turbidez & $0,694^{* *}$ & 0,471 \\
pH & $-0,480$ & $-0,023$ \\
Oxigênio dissolvido & $0,499^{*}$ & $-0,719^{* *}$ \\
Velocidade da correnteza & 0,221 & $-0,714^{* *}$ \\
Condutividade específica & $-0,732^{* *}$ & $0,515^{*}$ \\
Profundidade & $0,634^{* *}$ & $0,501^{*}$ \\
\hline
\end{tabular}




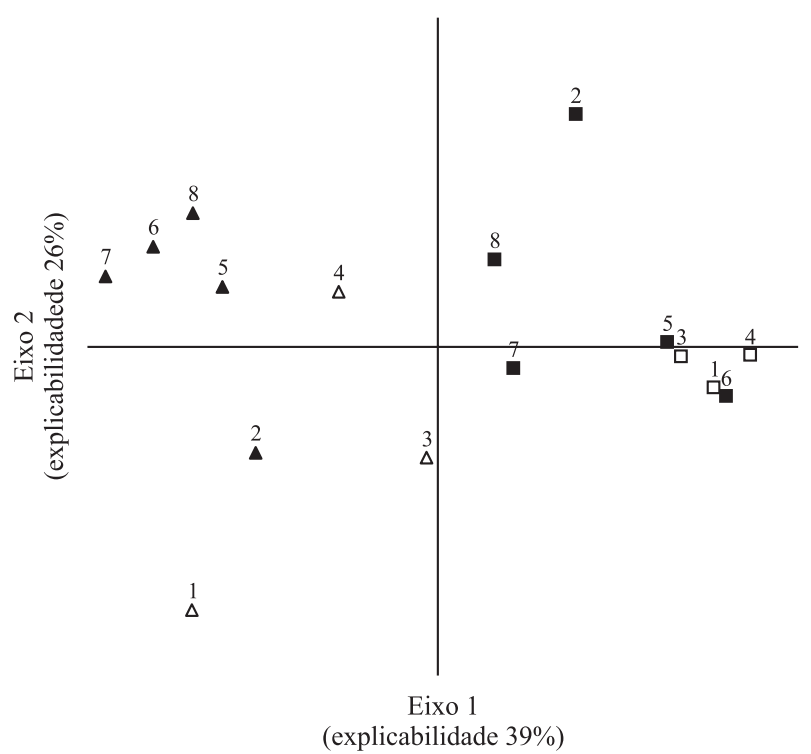

Figura 2. Análise dos Componentes Principais (PCA) dos pontos de amostragem da Serra da Prata com base nas variáveis ambientais (triângulos = inverno; quadrados = verão; brancos $=$ sombreados; pretos $=$ fortemente sombreados $)$.

Figure 2. Principal Component Analysis (PCA) scatter plot of the Serra da Prata sampling sites based on the environmental variables (triangles $=$ winter; squares $=$ summer; white $=$ shaded; black = strongly shaded).

mostrou que existiu diferença significativa entre eles $(z=2,52, P<0,05)$.

Ao contrário do observado no resultado da PCA, com base nas variáveis físico-químicas, a Análise de Correspondência Destendenciada (DCA) (tabela 5, figura 3) baseada na presença/ausência de espécies não revelou nenhuma tendência de agrupamento dos pontos de amostragem por estação do ano. Porém, nesta análise foi observada a separação pelo Eixo 1 dos pontos de amostragem sombreados dos fortemente sombreados.

Em adição ao resultado observado pela DCA, a Análise de Espécies Indicadoras (ISA) revelou a existência de duas espécies indicadoras, uma para ambientes fortemente sombreados (Microcoleus subtorulosus, $P<0,01)$ e outra para ambientes sombreados (Ecballocystis pulvinata var. pulvinata, $P<0,05$ ).

\section{Discussão}

O número global de táxons encontrados (14) pode ser considerado muito baixo comparado com outros estudos (29 táxons - Necchi Júnior et al. 2008, 30 Necchi Júnior et al. 2003, 31 - Necchi Júnior et al. 1994,
Tabela 5. Resultados da Análise de Correspondência Destendenciada (DCA) pela presença/ ausência das espécies e correlação de cada espécie com os dois primeiros eixos. (Valores de $r:<0,05^{*} ;<0,01^{* *} ;<0,001^{* * *}$ ).

Table 5. Detrended Correspondence Analysis (DCA) by species presence/ absence and correlation of each species with the first two axes. (Values of $r:<0,05^{*} ;<0,01^{* *}$; $\left.<0,001^{* * *}\right)$.

\begin{tabular}{lll}
\hline Espécies/ correlações & Eixo 1 & Eixo 2 \\
\hline Blennothrix komarekii & $-0,233$ & 0,102 \\
Microcoleus subtorulosus & $-0,550^{*}$ & $-0,671^{* *}$ \\
Nostoc verrucosum & $0,807^{* * *}$ & $-0,034$ \\
Nostochopsis lobatus & $-0,185$ & $-0,281$ \\
Phormidium aerugineo- & $-0,241$ & $-0,058$ \\
$\quad$ caeruleum & & \\
Phormidium retzii & 0,509 & $-0,023$ \\
Pleurocapsa fluviatilis & $-0,134$ & $-0,069$ \\
Ecballocystis pulvinata var. & $0,677^{* *}$ & $-0,044$ \\
$\quad$ pulvinata & & \\
Spirogyra sp. & 0,420 & $-0,023$ \\
Zygnema sp. & 0,157 & $-0,024$ \\
Batrachospermum ambiguum & $-0,185$ & $-0,281$ \\
Estágio “Chantransia” & $-0,290$ & $0,883^{* * *}$ \\
\hline
\end{tabular}

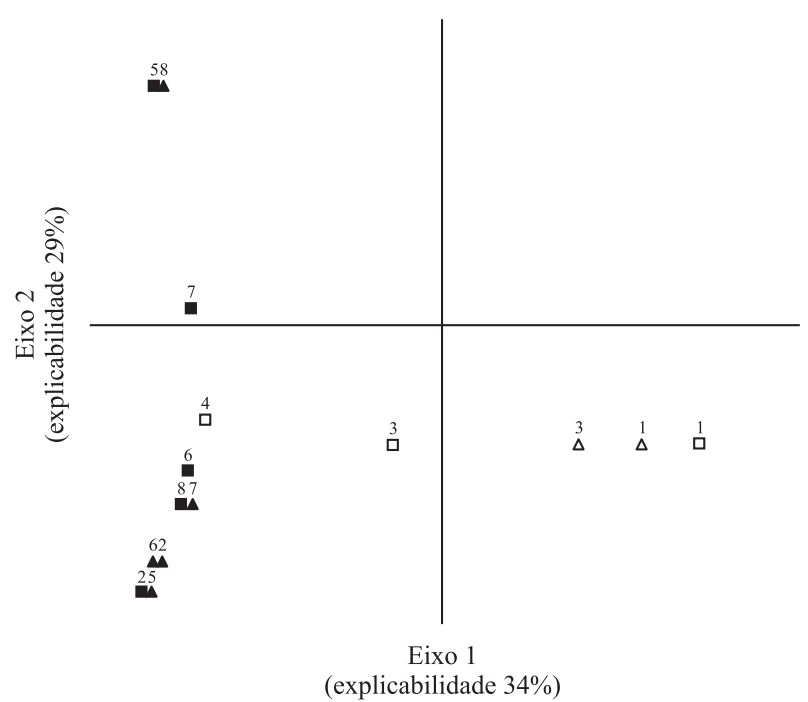

Figura 3. Análise de Correspondência Destendenciada (DCA) dos pontos de amostragem da Serra da Prata baseada na presença/ausência das macroalgas (triângulos = inverno; quadrados $=$ verão; brancos $=$ sombreados; pretos $=$ fortemente sombreados).

Figure 3. Detrended Correspondence Analysis (DCA) scatter plot of the Serra da Prata sampling sites based on the presence/ absence of macroalgae (triangles $=$ winter; squares = summer; white $=$ shaded; black $=$ strongly shaded) . 
35 - Krupek et al. 2007, 37 - Branco \& Necchi Júnior 1998, 42 - Branco \& Necchi Júnior 1996). Entretanto, os valores de riqueza média por ponto de amostragem ( $\bar{x}=3,0 \pm 0,9$ táxons) encontrados no presente estudo estiveram dentro do padrão recorrentemente encontrado em ecossistemas lóticos (p. ex. 1,3 $\pm 1,2$ - Sheath \& Müller 1997; 2,5 \pm 1,6 - Branco \& Necchi Júnior 1996; 2,6 $\pm 1,5$ - Krupek et al. 2007; 2,6 $\pm 1,6$ - Branco et al. 2009; 4,2 \pm 2,3 - Necchi Júnior et al. 2008 e; 6,7 \pm 2,7 - Necchi Júnior et al. 2003).

A abundância nos pontos de amostragem apresentou valores extremamente baixos $(\bar{x}=1,4 \pm 1,2$ de cobertura percentual do leito por amostragem) quando comparado com outros estudos (p. ex. 5,2 $\pm 8,4$ - Krupek et al. 2007; 5,7 \pm 7,4 - Branco et al. 2009; 13,5 $\pm 11,9-$ Necchi Júnior et al. 2008 e; 15,4 $\pm 21,0$ - Branco \& Necchi Júnior 1996).

A maioria dos táxons encontrados na Serra da Prata (64\%) ocorreram em um único ponto de amostragem. Esta tendência de alta proporção de táxons de ocorrência restrita a um ou poucos segmentos tem sido freqüentemente encontrada na literatura tanto para o Brasil quanto para outras regiões do mundo. Por exemplo, 49\% dos táxons reportados por Branco \& Necchi Júnior (1996) para a Mata Atlântica do Estado de São Paulo, 58\% por Krupek et al. (2007) em uma bacia de drenagem no centro-sul do Paraná, 52\% por Branco et al. (2009) na região centro-oeste do Paraná e, $55 \%$ por Necchi Júnior et al. (2008) no Parque Nacional de Itatiaia ocorreram em somente um único ponto de amostragem. Da mesma forma, Sheath \& Burkholder (1985) e Sheath et al. (1986, 1989) encontraram entre 44 a $55 \%$ do total de espécies ocorrendo em menos que $5 \%$ dos pontos de amostragem em estudos realizados na América do Norte. Adicionalmente, Hu \& Xie (2006) para riachos na China, reportaram $28 \%$ de táxons com ocorrência restrita. Esses achados confirmam que a ocorrência restrita pode ser um padrão geral característico para macroalgas de ambientes lóticos. Por outro lado, o padrão de distribuição em mosaico e a dominância por poucas espécies, considerado como sendo universal (Necchi Júnior et al. 2008) para comunidades de macroalgas de riacho não foi claramente observado no presente estudo.

A Análise de Correspondência Destendenciada (DCA), revelou que a estrutura das comunidades de macroalgas não seguiu o mesmo padrão encontrado na PCA das variáveis físicas e químicas, onde os pontos foram agrupados pela época de amostragem (inverno ou verão). Mesmo a precipitação, que é recorrentemente sugerida como fator chave na definição dos padrões de distribuição temporal das macroalgas lóticas (Necchi Júnior et al. 1991, Necchi Júnior \& Pascoaloto 1993, Branco \& Necchi Júnior 1997), não revelou nenhuma clara influência sobre as comunidades estudadas.

Este resultado sugere que as variáveis físicas e químicas mensuradas podem ser pouco representativas para explicar o padrão de distribuição espacial das macroalgas na região de estudo. Resultados similares também foram encontrados em estudos semelhantes (e.g. Necchi Júnior et al. 1991, 2000, Branco \& Necchi Júnior 1996, 1998, Sherwood \& Sheath 1999, Sherwood et al. 2000, Verb \& Vis 2001, Hu \& Xie 2006, Branco et al. 2009).

Desta forma, os resultados aqui encontrados, particularmente o fato da maior parte das espécies apresentarem ocorrência restrita a um ou poucos pontos de amostragem, levam a supor que as características locais podem ser extremamente relevantes na determinação da estrutura das comunidades de macroalgas da Serra da Prata. Diante de resultados semelhantes, Krupek et al. (2007) e Branco et al. $(2009,2008)$ estudando riachos nas regiões centro-sul e centro-oeste do Paraná, propõem que a heterogeneidade espacial de ambientes lóticos e a combinação dessas variáveis em cada fragmento ("mancha") do riacho em um dado tempo, são os fatores que definem a diversidade e a distribuição espacial e temporal nestes ambientes.

Neste sentido, avaliações do efeito da heterogeneidade das características físicas e químicas tanto em meso (centimétrica a métrica) quanto em microescala (milimétrica a centimétrica), da freqüência e intensidade de distúrbios, dos mecanismos de colonização e dos impactos de herbívoros são fortemente sugeridas em estudos futuros.

Embora, a DCA não tenha revelado a formação de grupos com base nas variáveis físicas e químicas, esta análise revelou a existência de comunidades específicas para ambientes sombreados e fortemente sombreados. A possível relação entre as comunidades de macroalgas e o nível de sombreamento dos ambientes lóticos da Serra da Prata observada na DCA foi corroborada pela ISA. Tais resultados refletem uma tendência que aponta a habilidade das Cyanobacteria de viverem em ambientes com limitação de luz (Sheath \& Burkholder 1985, Branco \& Necchi Júnior 1998) e o melhor desenvolvimento das algas verdes em ambientes com maior disponibilidade de luz (Sheath \& Burkholder 1985, Biggs \& Price 1987, Okada \& Watanabe 2002). Este conjunto de resultados sugere que em grande escala (entre segmentos dentro da área de estudo) o único fator relacionado com a distribuição ecológica das comunidades de macroalgas 
da Serra da Prata foi o sombreamento provocado pela vegetação marginal.

Agradecimentos - Os autores agradecem à gerência do Parque Nacional Saint-Hilaire/Lange pelo apoio durante os trabalhos de campo, a Capes pela bolsa de mestrado concedida ao primeiro autor e a Fundação de Amparo à Pesquisa do Estado de São Paulo (Fapesp), Auxílio à Pesquisa (Proc. 04/09996-2), pelo apoio financeiro durante a execução dos trabalhos de campo.

\section{Referências Bibliográficas}

ALLAN, J.D. 1995. Stream ecology: struture and function of running waters. Chapman \& Hall, London.

BIGGS, B.J.F. \& PRICE, G.M. 1987. A survey of filamentous algal proliferations in New Zealand rivers. New Zealand Journal of Marine and Freshwater Research 21:175-19.

BORGES, F.R. \& NECCHI JÚNIOR, O. 2006. Patterns of spatial distribution in macroalgal communities from tropical lotic ecosystems. Revista Brasileira de Botânica 29:669-680.

BRANCO, C.C.Z.\& NECCHI JÚNIOR, O. 1996. Distribution of stream macroalgae in the eastern Atlantic Rainforest of São Paulo State, sotheastern Brazil. Algological Studies 333:139-150.

BRANCO, C.C.Z., KRUPEK, R.A. \& PERES, C.K. 2008. Seasonality of macroalgal communities in a subtropical drainage basin in Paraná State, Southern Brazil. Brazilian Journal of Biology 68:631-637.

BRANCO, C.C.Z., KRUPEK, R.A. \& PERES, C.K. 2009. Ecological distribution of stream macroalgal communities from mid-western region of Paraná State, Southern Brazil: evidence of the importance of local scale variation. Brazilian Archives of Biology and Technology 52:379-386.

BRANCO, L.H.Z. \& NECCHI JÚNIOR, O. 1997. Seasonality of macroalgae in three tropical drainage basins in São Paulo State, southeastern Brazil. Archiv für Hydrobiologie 141:75-91.

BRANCO, L.H.Z. \& NECCHI JÚNIOR, O. 1998. Distribution of macroalgae in three tropical drainage basins of southeastern Brazil. Archiv für Hydrobiologie 142:241-256.

CONOVER, W.J. 1971. Practical nonparametric statistics. John Wiley \& Sons, New York.

DENICOLA, D.M., HOGLAND, K.D. \& ROEMER, S.C. 1992. Influence of canopy cover on spectral irradiance and periphyton assemblages in a prairie stream. Journal of the North American Benthological Society 11:391-404.

DIGBY, P.G.N. \& KEMPTON, R.A. 1987. Multivariate analysis of ecological communities. Chapman \& Hall, London.
DUFRÊNE, M. \& LEGENDRE, P. 1997. Species assemblages and indicator species: the need for a flexible asymmetrical approach. Ecological Monographs 67:345-366.

ENTWISLE, T.J. 1990. Macroalgae in the upper Yarra \& Watts River catchments: distribution \& phenology. Australian Journal of Marine and Freshwater Research 41:505-522.

GORDON, N.D., MCMAHON, T.A. \& FINLAYSON, B.L. 1992. Stream hydrology: an introduction for ecologists. John Wiley \& Sons, Chichester.

HU, B.F. \& XIE, S.L. 2006. Effect of seasonality on distribution of macroalgae in a stream system (Xin'an Spring) in Shanxi Province, North China. Journal of Integrative Plant Biology 48:889-896.

KRUPEK, R.A., BRANCO, C.C.Z. \& PERES, C.K. 2007. Distribuição ecológica das comunidades de macroalgas da bacia de drenagem do Rio das Pedras, região centrosul do Estado do Paraná, Sul do Brasil. Revista Brasileira de Botânica 30:173-182.

LEUKART, P. 1995. Studies on the macroalgal vegetation of a small soft-water stream in the Spessart mountains, Germany, with reference to algal distribution and seasonality. Algological Studies 79:77-92.

MAACK, R. 1981. Geografia física do Estado do Paraná. $2^{\mathrm{a}}$ ed. Editora José Olympio, Rio de Janeiro.

MCCUNE, B. \& MEFFORD, M.J. 1999. Multivariate analysis of ecological data. MjM Software, Glendem Beach.

NECCHI JÚNIOR, O. 1993. Distribution and seasonal dynamics of Rhodophyta in the Preto River basin, southeastern Brazil. Hydrobiologia 250:81-90.

NECCHI JÚNIOR, O. \& PASCOALOTO, D. 1993. Seasonal dynamics of macroalgal communities in the Preto River basin, São Paulo, southeastern Brazil. Archiv für Hydrobiologie 129:231-252.

NECCHI JÚNIOR, O., DIP, M.R. \& GÓES, R.M. 1991. Macroalgae of a stream in southeastern Brazil: composition, seasonal variation and relation to physical and chemical variables. Hydrobiologia 213:241-250.

NECCHI JÚNIOR, O., PASCOALOTO, D. \& BRANCO, L.H.Z. 1994. Distribution of macroalgae in a tropical river basin from southeastern Brazil. Archiv für Hydrobiologie 129:459-471.

NECCHI JÚNIOR, O., BRANCO, L.H.Z. \& BRANCO, C.C.Z. 1995. Comparison of three techniques for estimating periphyton abundance in bedrock streams. Archiv für Hydrobiologie 134:393-402.

NECCHI JÚNIOR, O., BRANCO, C.C.Z. \& BRANCO, L.H. Z. 2000. Distribution of stream macroalgae in São Paulo State, southeastern Brazil. Algological Studies 97:43-57.

NECCHI JÚNIOR, O., BRANCO, L.H.Z. \& BRANCO, C.C.Z.2003. Ecological distribution of stream macroalgal communities from a drainage basin in the Serra da Canastra National Park, Minas Gerais, Southeastern Brazil. Brazilian Journal of Biology 63:1-12. 
NECCHI JÚNIOR, O., BRANCO, L.H.Z. \& SPEZAMIGLIO, D.N. 2008. Distribuição ecológica de comunidades de macroalgas de ambientes lóticos do Parque Nacional de Itatiaia (RJ, MG) Brasil. Revista Brasileira de Botânica 31:135-145.

OKADA, H. \& WATANABE, Y. 2002. Effect of physical factors on the distribution of filamentous green algae in the Tama River. Limnology 3:121-126.

PFISTER, P. 1993. Seasonality of macroalgal distribution patterns within the reach of a gravel stream (Isar, Tyron, Austria). Archiv für Hydrobiologie 129: 89-107.

PRÓ-ATLÂNTICA. 2002. Zoneamento ecológicoeconômico e plano de gestão da APA de Guaratuba. Relatório de consultoria apresentado por Harald Müeller, Curitiba.

RODERJAN, C.V., GALVÃO, F., KUNIYOSHI, Y.S. \& HATSCHBACH, G.G. 2002. As unidades fitogeográficas do Estado do Paraná. Ciência \& Ambiente 24:75-92.

SHEATH, R.G. \& BURKHOLDER, J. 1985. Characteristics of softwater stream in Rhode Island. II: Composition and seasonal dynamics of macroalgae communities. Hydrobiologia 128:109-118.
SHEATH, R.G. \& COLE, K.M. 1992. Biogeography of stream macroalgae in North America. Journal of Phycology 28:448-460.

SHEATH, R.G. \& MÜLLER, K.M. 1997. Distribution of stream macroalgae in four high artic drainage basins. Artic 50:355-364.

SHEATH, R.G., MORISON, M.O., KORCH, J.E., KACZMARCZYK, D. \& COLE, K.M. 1986. Distribution of stream macroalgae in south-central Alaska. Hydrobiologia 135:259-269.

SHEATH, R.G., HAMILTON, P.B., HAMBROOK, J.A. \& COLE, K.M. 1989. Stream macroalgae of eastern boreal forest region of North America. Canadian Journal of Botany 67:3553-3562.

SHERWOOD, A.R., RINTOUL, T.L., MÜLLER, K.M. \& SHEATH, R.G. 2000. Seasonality of epilithic diatoms, macroalgae and macrophytes in a spring-fed stream system in Ontario, Canada. Hydrobiologia 435:143-152.

SHERWOOD, A.R. \& SHEATH, R.G. 1999. Seasonality of macroalgae and epilithic diatoms in spring-fed streams in Texas, U.S.A. Hydrobiologia 390:73-82.

VERB, R.G. \& VIS, M.L. 2001. Macroalgal communities from a acid mine drainage impacted watershed. Aquatic Botany 71:93-107. 